\title{
A cross-species analysis of the aversiveness of denatonium saccharide and quinine
}

\author{
STEPHEN F. DAVIS, KIMBERLY J. HOSKINSON, KYLE A. WILDER, \\ JULIE A. SANDER, R. KURT LARSEN, and MEGAN KNAPP \\ Emporia State University, Emporia, Kansas
}

\begin{abstract}
Although a recent report (Davis, Grover, \& Erikson, 1987) indicated that humans perceive denatonium saccharide as significantly more bitter than quinine, Davis, Grover, Erickson, Miller, and Bowman (1987) indicated that albino laboratory rats perceive quinine as the more bitter substance. The present investigation sought to extend the generality of the latter finding to two additional species. The results of two-bottle preference tests using several concentrations of these bitter substances with prairie dogs and gerbils corresponded more closely to the laboratory rat data than to the human data.
\end{abstract}

In agreement with the contention that the recently discovered chemical denatonium saccharide is the most bitter substance currently available, recent reports have demonstrated that denatonium presentation significantly suppressed fluid intake in rats (Davis et al., 1986) and grasshopper mice (Langley, Theis, Davis, Richard, \& Grover, 1987). On the other hand, Davis, Grover, Erickson, Miller, and Bowman (1987) directly compared denatonium and the more common bitter, quinine. This experiment demonstrated that albino laboratory rats consumed significantly greater amounts of denatonium than quinine. Furthermore, unlike quinine, denatonium was unable to support negative hedonic shifts when paired with neutral flavors. These results, in conjunction with data showing that the suppressive effects of denatonium may be rather transitory (Davis et al., 1986), suggest that the initial claims of denatonium's excessive bitterness may have been exaggerated. However, it is worth noting that the data questioning the strength of denatonium's suppressive power came from studies in which rodents were used as subjects.

After asking humans to compare the aversiveness of denatonium and quinine, Davis, Grover, and Erickson (1987) reported a pattern of results that was radically different. These investigators found that independent groups rated denatonium as being significantly more bitter than quinine at three different concentrations: 1 part chemical per $10,000,5,000$, or 1,000 parts water.

Through the testing of two additional species, Mongolian gerbils and black-tailed prairie dogs, the present study sought to provide some resolution to these discrepant data. These species were selected for testing because of availability, and because they potentially represented a more direct link with animals in a more natural state. Possibly generations of inbreeding have produced in the al-

Address correspondence to Stephen F. Davis, Department of Psychology, Emporia State University, 1200 Commercial, Emporia, KS 66801-5087. bino laboratory rat an organism with atypical responsivity to the two bitters in question.

\section{METHOD}

\section{Subjects}

A total of 24 Mongolian gerbils (Meriones unquiculatus) and 4 blacktailed prairie dogs (Cynomys ludovicianus) served as subjects. Of the 24 gerbils, 19 ( 5 males, 14 females) participated in the entire experiment; the remaining 5 gerbils ( 2 males, 3 females) were tested only in Phase 3, the final phase of the study. All 4 prairie dogs $(1 \mathrm{male}, 3 \mathrm{fe}-$ males) were tested during all three phases. The gerbils ranged in age from 60 to 120 days of age. Although the ages of the prairie dogs were not known, all clearly were adults.

\section{Apparatus}

All testing took place in the home cages, which were polypropylene mouse breeding cages for the gerbils and glass aquariums for the prairie dogs. Food was available on a free-feeding basis for all subjects for the duration of the experiment.

\section{Procedure}

The three phases of experimental testing encompassed a total of 37 days. Phase 1 (20 days) constituted a denatonium versus water preference test. In blocks of 5 days each, the animals were allowed to consume plain tap water or a solution of 1 part denatonium per 20,000 , $10,000,5,000$, or 1,000 parts water, respectively.

Phase 2 (5 days) was designed to provide exposure to quinine for those animals that had participated in the denatonium tests of Phase 1 . Such exposure was deemed necessary to preclude a neophobic response to the quinine when it was introduced in Phase 3. The solution used during Phase 2 was 1 part quinine per 10,000 parts water.

During Phase 3, which lasted 12 days, all subjects received simultaneous access to similar concentrations of denatonium and quinine. The three concentrations employed were 1 part chemical per 10,000,5,000, and 1,000 parts of water. Testing was begun with the most dilute, 1 part per 10,000 parts water, solution. After 4 days of testing, the next strongest solution was substituted until all three solutions had received 4 days of testing.

All fluids were presented in 50-ml polypropylene centrifuge tubes fitted with spill-resistent sippers. The subjects were given free access to fluids during all phases of the experiment. Fluid consumption was recorded daily at $1600 \mathrm{~h}$. Fluid levels were replenished at this time.

\section{RESULTS}

Group mean consumption (in milliliters) of denatonium saccharide and water for the prairie dogs for each of the 
four concentrations employed in Phase 1 is shown in Figure 1. As can be seen, an initial preference for denatonium at the weakest concentration (1 part per 20,000 parts water) was gradually reversed, and a strong preference for water was established as the concentration of denatonium was increased to 1 part per 1,000. Analysis of these data yielded significance for fluid $[F(1,13)=20.808$, $p=.018]$, days $[F(5,12)=7.525, p=.003]$, and days $\times$ concentration $[F(12,36)=2.051, p=.047]$ effects. Further inspection of the significant interaction through the use of Newman-Keuls tests indicated that denatonium and water were consumed in equal amounts at the 1 part per 20,000 and 1 part per 10,000 concentrations, whereas water was preferred significantly $(p<.05)$ at the 1 part per 5,000 and 1 part per 1,000 concentrations.

Group mean consumption for the gerbils during Phase 1 is shown in Figure 2. The gerbils also displayed an initial preference for denatonium that was gradually reversed to a water preference by the end of the phase. Analysis of these data yielded significance for the fluid $[F(1,19)=$ $7.381, p=.013]$, days $[F(4,76)=2.515, p=.047]$, fluid $\times$ concentration $[F(3,57)=7.647, p<.001]$, concentration $\times$ days $[F(12,228)=3.284, p<.001]$ and fluid $\times$ concentration $\times$ days $[F(12,228)=2.263$, $p=.010$ ] effects. Newman-Keuls tests, used to probe the significant three-way interaction, indicated that significantly $(p<.05)$ more denatonium than water was consumed on Days 1-3 at the 1 part per 20,000 concentration. On the other hand, significantly $(p<.05)$ more

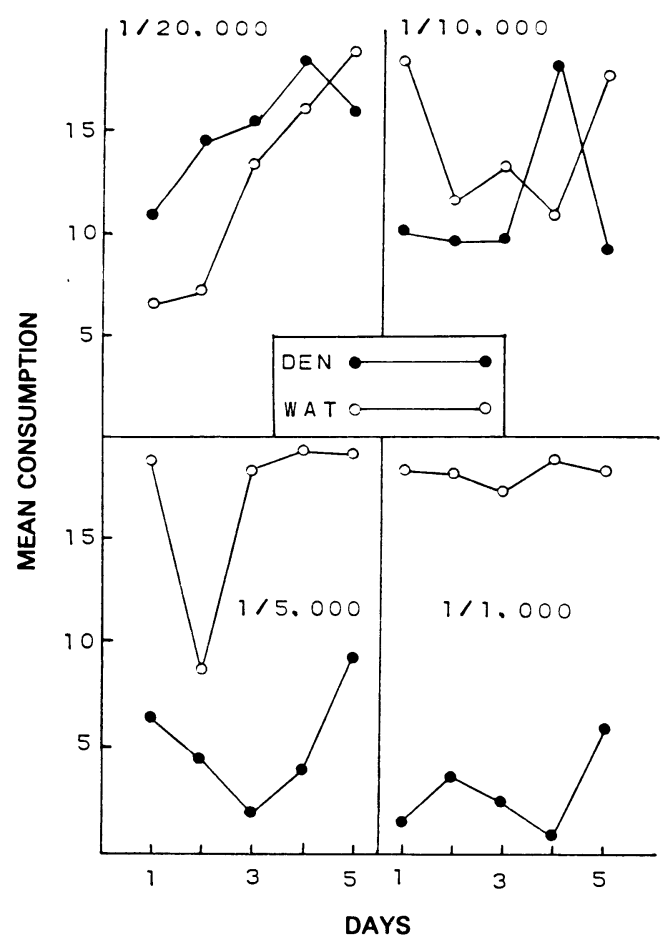

Figure 1. Mean denatonium saccharide and water consumption (in milliliters) shown by the prairie dogs during Phase 1.

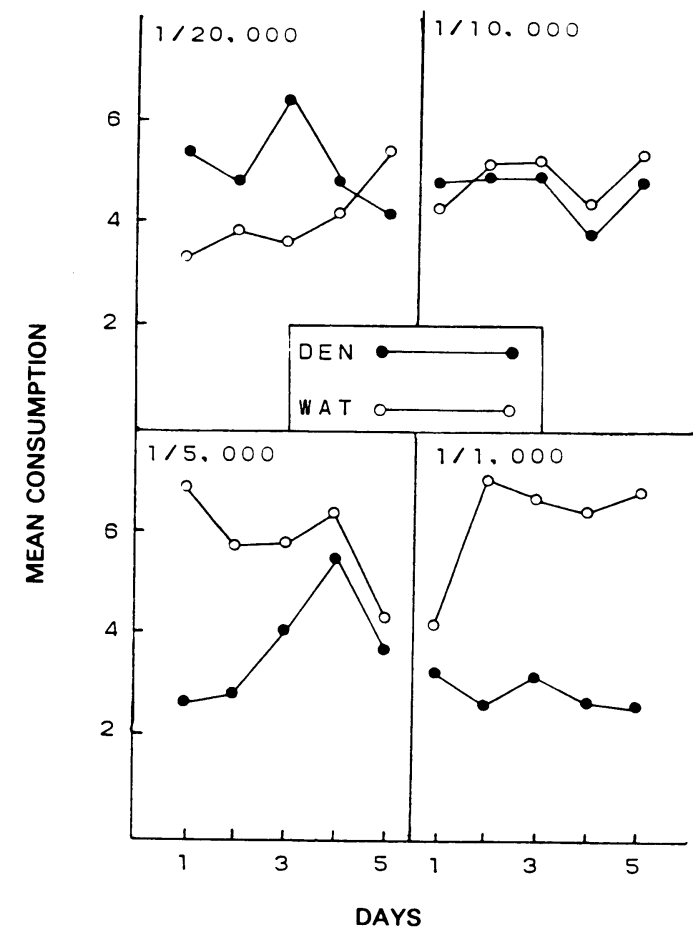

Figure 2. Mean denatonium saccharide and water consumption (in milliliters) shown by the gerbils during Phase 1.

water than denatonium saccharide was consumed on Days 1-3 for the 1 part per 5,000 concentration and on Days 2-5 for the 1 part per 1,000 concentration.

Figure 3 displays mean quinine and water consumption for the prairie dogs and gerbils during Phase 2, that is, quinine habituation. Because analysis of the consumption data of the gerbils failed to yield significant effects, it can be concluded that these subjects failed to display a significant fluid preference during this phase. On the other hand, a significant fluid effect $[F(1,3)=14.037$, $p=.031]$ supported the graphic impression that the prairie dogs preferred water over quinine.

Finally, Figure 4 depicts the results of the quinine versus denatonium tests conducted during Phase 3. Clearly, it is not as easy to generate uniform statements concerning the reactions of the three groups to these two flavors. For example, the prairie dogs appeared to prefer denatonium at the two weaker concentrations, but reversed their preference at the strongest concentration. A significant fluid $\times$ concentration interaction $[F(2,6)=13.740$, $p=.006]$ supported this graphic impression. Some, albeit limited, preference for denatonium also was noted for the gerbils that had experienced the Phase 1 and Phase 2 treatments, that is, the trained gerbils. The presence of a significant fluid $\times$ concentration $\times$ days interaction $[F(6,108)=2.370, p=.034]$ underscored the tenuousness of attempting to make a generalization concerning this group. Additionally, the concentration $[F(2,36)=5.787, p=.006]$, fluid $\times$ concentration 


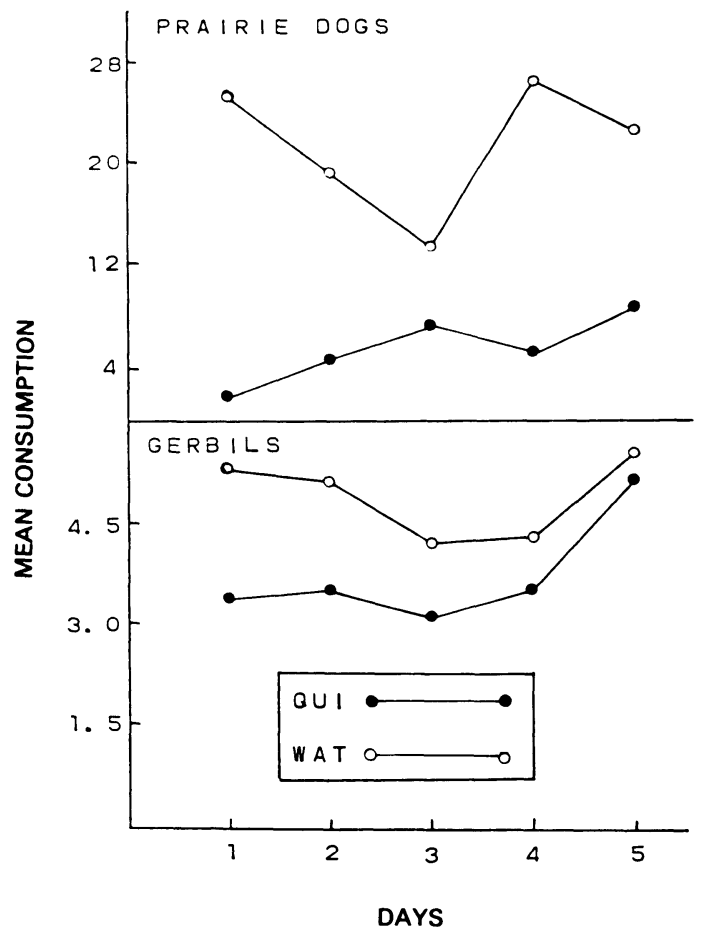

Figure 3. Mean quinine and water consumption (in milliliters) for the prairie dogs and gerbils during Phase 2.

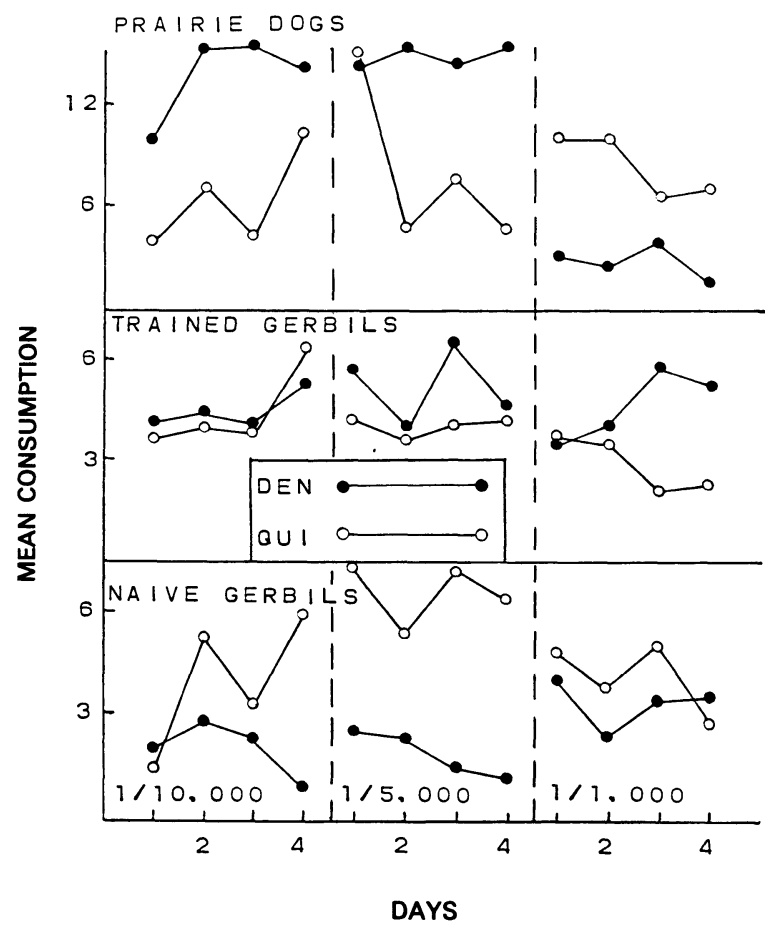

Figure 4. Mean consumption of denatonium saccharide and quinine (in milliliters) by the prairie dog (top panel), the gerbils tested during Phases 1 and 2 (middle panel), and the gerbils tested only during Phase 3 (bottom panel).
$[F(2,36)=7.675, p=.002]$, days $[F(3,54)=2.934$, $p=.04]$, and concentration $\times$ days $[F(6,108)=6.806$, $p<.001]$ effects achieved significance. Inspection of the significant three-way interaction indicated that the trained gerbils consumed significantly $(p<.05)$ more denatonium than quinine on Days 1 and 3 of 1 part per 5,000 training and on Days 3 and 4 of 1 part per 1,000 training.

Turning to the performance of the naive gerbils during Phase 3 testing, it appears that these animals, unlike the other two groups, had a preference for quinine. Analysis of these data yielded significance for the concentration $[F(2,8)=10.634, p=.006]$ and fluid $\times$ concentration $[F(2,8)=12.583, p=.003]$ effects. Corroborating the graphic impression, it was found that these animals consumed significantly $(p<.01)$ more quinine than water during the 1 part per 5,000 testing.

\section{DISCUSSION}

What conclusions are prompted by the present data? First, in agreement with the initial studies using laboratory rats, both the prairie dogs and gerbils failed to find the 1 part per 20,000 and 1 part per 10,000 concentrations of denatonium saccharide aversive. Only at the most extreme concentrations was a preference for water displayed. Clearly, these results are not in agreement with the human data reported by Davis, Grover, and Erickson (1987) in which all concentrations of denatonium saccharide were rated as quite aversive. Based upon the data gathered to date, it can be concluded that rodents simply do not perceive denatonium saccharide as aversive as do human subjects. Hence, if one is planning to employ denatonium saccharide as a deterrent, then a wide range of concentrations potentially may be employed with humans, whereas only the strongest concentrations should be employed with rodents.

Second, it is interesting to note that the gerbils failed to display an aversion to quinine during Phase 2 . This suggests that, unlike the laboratory rats tested by Davis, Grover, Erickson, et al. (1987), this species does not find quinine especially aversive. On the other hand, the prairie dogs found the 1 part per 10,000 solution of quinine to be aversive. Hence, a generalization concerning the aversiveness of quinine among rodent species does not seem advisable at this point.

The inability to make such a generalized statement perseverated into Phase 3 when denatonium saccharide and quinine were directly compared. Unlike the laboratory rats tested by Davis et al. (1987), the prairie dogs and trained gerbils found quinine to be less aversive than denatonium saccharide at the 1 part per 1,000 concentration.

Finally, the reaction of the naive gerbils to quinine and denatonium saccharide during Phase 3 is worthy of comment. That these animals displayed a significant preference for quinine at the 1 part per 5,000 concentration, and that the trained gerbils displayed the opposite pattern, indicates that prior exposure to these chemicals may have an influence upon subsequent preferences and/or sensitivities. Such speculations notwithstanding, it is clear that these animals also failed to display a strong aversion to quinine as did the laboratory rats. Yet, they also failed to have a significant aversion to denatonium saccharide at the strongest concentration.

Clearly, the present results are supportive of the previous animal data in indicating that denatonium saccharide, despite being 3,000 times more bitter than quinine, is not perceived in this manner by two rodent species. Whereas denatonium saccharide is a potent deterrent to human consumption, the accumulating rodent data point to the conclusion that its use may be little more than an oddity in the animal laboratory.

\section{REFERENCES}

Davis, S. F., Cunningham, L. A., Burke, T. J., Richard, M. M., LANGLEY, W. M., \& TheIs, J. (1986). A preliminary analysis of the 
suppressive effects of denatonium saccharide. Bulletin of the Psychonomic Society, 24, 229-232.

Davis, S. F., Grover, C. A., E Erickson, C. A. (1987). A comparison of the aversiveness of denatonium saccharide and quinine in humans. Bulletin of the Psychonomic Society, 25, 462-463.

Davis, S. F., Grover, C. A., Erickson, C. A., Miller, L. A., \& Bowman, J. A. (1987). Analyzing the aversiveness of denatonium saccharide and quinine in rats. Perceptual \& Motor Skills, 64, 1215-1222.
Langley, W. M., Theis, J., Davis, S. F., Richard, M. M., \& Grover, C. A. (1987). Effects of denatonium saccharide on the drinking behavior of the grasshopper mouse (Onychomys leucogaster). Bulletin of the Psychonomic Society, 25, 17-19.

(Manuscript received for publication February 18, 1988.) 